\title{
Subradiance in a Large Cloud of Cold Atoms
}

\author{
William Guerin,, ${ }^{1, *}$ Michelle O. Araújo, ${ }^{1,2}$ and Robin Kaiser ${ }^{1}$ \\ ${ }^{1}$ Institut Non Linéaire de Nice, CNRS and Université Nice Sophia Antipolis, 1361 route des Lucioles, O6560 Valbonne, France \\ ${ }^{2}$ CAPES Foundation, Ministry of Education of Brazil, Brasília DF 70040-020, Brazil
}

(Received 8 September 2015; published 22 February 2016)

\begin{abstract}
Since Dicke's seminal paper on coherence in spontaneous radiation by atomic ensembles, superradiance has been extensively studied. Subradiance, on the contrary, has remained elusive, mainly because subradiant states are weakly coupled to the environment and are very sensitive to nonradiative decoherence processes. Here, we report the experimental observation of subradiance in an extended and dilute cold-atom sample containing a large number of particles. We use a far detuned laser to avoid multiple scattering and observe the temporal decay after a sudden switch-off of the laser beam. After the fast decay of most of the fluorescence, we detect a very slow decay, with time constants as long as 100 times the natural lifetime of the excited state of individual atoms. This subradiant time constant scales linearly with the cooperativity parameter, corresponding to the on-resonance optical depth of the sample, and is independent of the laser detuning, as expected from a coupled-dipole model.
\end{abstract}

DOI: 10.1103/PhysRevLett.116.083601

Despite its many applications, ranging from astrophysics [1] to mesoscopic physics [2,3] and quantum information technology [4], light interacting with a large ensemble of $N$ scatterers still bears many surprising features and is at the focus of intense research. For $N=2$ atoms placed close together, the in-phase oscillation of the induced dipoles produces a large, superradiant dipole, whereas the out-ofphase oscillation corresponds to a subradiant quadrupole. Generalizing for $N \gg 2$, Dicke has shown that, for samples of a small size compared to the wavelength of the atomic transition, the symmetric superposition of atomic states induces superradiant emission, scaling with the number of particles $N$, whereas the antisymmetric superpositions are decoupled from the environment, with vanishing emission rates, which corresponds to subradiance [5].

Dicke superradiance has been extensively studied in the 1970s [6-8] but the observation of its counterpart, subradiance, has been restricted to indirect evidence of modified decay rates in one particular direction [9] or in systems of two particles at very short distance [10-12]. One challenge for the observation of subradiance by a large number of particles is the fragile nature of these states, which require protection from any local nonradiative decay mechanism [13]. Furthermore, contrary to the two-atom case, for which the distance between atoms has to be small compared to the wavelength, for $N \gg 2$, the retarded, long-range resonant dipole-dipole interaction [14] gives rise to super- and subradiant effects ("cooperative scattering") also in dilute samples, with interatomic distances much larger than the wavelength, and corresponding large system sizes. Since, for $N>2$, the Hamiltonians for short and long-range interactions do not commute, the collective eigenstates due to the long-range interactions are suppressed by short-range interactions [8]. These short-range or near-field effects (or "van der Waals dephasing") thus need to be avoided in this case. As a consequence-and maybe counterintuitively - a large and dilute sample of interacting dipoles is the most appropriate system for the observation of $N$-body subradiance.

In this regime, and in the weak excitation limit ("single-photon superradiance") [15-17], it has been shown that the superradiant enhancement of the emission rate scales as the cooperativity parameter $N / M$, where $M$ is the number of available modes for the electromagnetic radiation [18-21]. For a spherical sample of radius $R$, $M \sim\left(k_{0} R\right)^{2}$, where $k_{0}=2 \pi / \lambda$, this cooperativity parameter is proportional to the peak on-resonant optical depth of the atomic cloud, given by $b_{0}=3 N /\left(k_{0} R\right)^{2}$ for a coldatom cloud with a Gaussian density distribution of rms radius $R$. This number can be large even at low density. In a recent work [22], we used a coupled-dipole model to generalize this result to subradiance (see also the Supplemental Material [23]). In this Letter, we report the experimental observation of subradiance in this weakexcitation, dilute- and extended-sample limit.

In our experiment, we load $N \approx 10^{9}{ }^{87} \mathrm{Rb}$ atoms from a background vapor into a magneto-optical trap (MOT) for $50 \mathrm{~ms}$. A compressed MOT (30 ms) period allows for an increased and smooth spatial density with a Gaussian distribution of $\mathrm{rms}$ size $R \approx 1 \mathrm{~mm}$ (typical density $\rho \approx 10^{11} \mathrm{~cm}^{-3}$ ) and a reduced temperature $T \approx 50 \mu \mathrm{K}$. We then switch off the MOT trapping beams and magnetic field gradient and allow for $3 \mathrm{~ms}$ of free expansion, used to optically pump all atoms into the upper hyperfine ground state $F=2$. Next, we apply a series of 12 pulses of a weak probe beam (waist $w=5.7 \mathrm{~mm}$ ), linearly polarized and detuned by $\delta=\left(\omega-\omega_{0}\right) / \Gamma$ from the closed atomic transition $F=2 \rightarrow F^{\prime}=3$. Here, $\omega$ is the frequency of the laser, $\omega_{0}$ the frequency of the atomic transition (of wavelength $\lambda=2 \pi c / \omega_{0}=780.24 \mathrm{~nm}$ ), and $\Gamma / 2 \pi=6.07 \mathrm{MHz}$ 


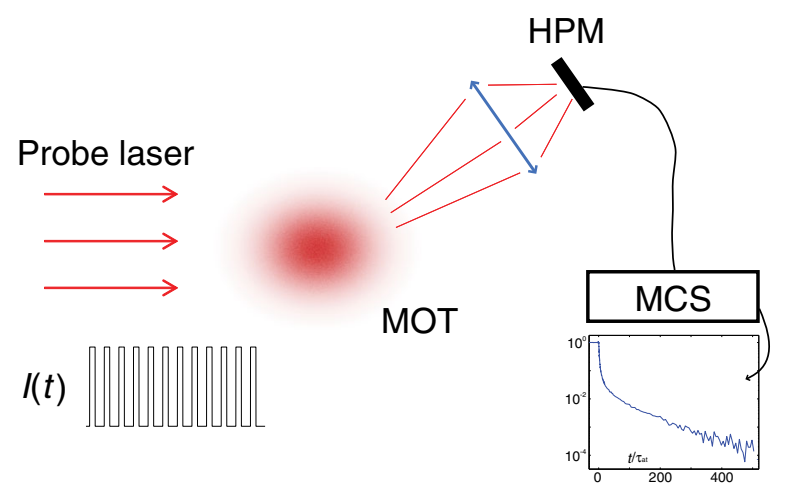

FIG. 1. Principle of the experiment. A large probe laser illuminates the atomic sample for $30 \mu \mathrm{s}$ and is switched off rapidly. The fluorescence at $\sim 35^{\circ}$ is collected by a hybrid photomultiplier (HPM) and recorded on a multichannel scaler (MCS). The experiment is repeated 500000 times. At each cycle, 12 pulses are recorded during the free expansion of the cloud, allowing the on-resonance optical depth to vary.

its linewidth. Note that when we varied the detuning, we also varied the laser intensity accordingly in order to keep the saturation parameter constant at $s \simeq 4.5 \times 10^{-2}$. The pulses of duration $30 \mu \mathrm{s}$ and separated by $1 \mathrm{~ms}$ are obtained by using two acousto-optical modulators in series to reach an extinction ratio better than $10^{-4}$. The $90 \%-10 \%$ fall time at the switch-off is $\sim 15 \mathrm{~ns}$, limiting the possibility of studying superradiance, but convenient for detecting subradiance. Between subsequent pulses of each series, the size of the cloud increases because of thermal expansion, and the atom number decreases due to off-resonant optical pumping into the $F=1$ hyperfine state during each pulse. The corresponding change of the on-resonant optical depth $b_{0}$ allows us to conveniently measure the decay of the fluorescence as a function of $b_{0}$ and investigate whether $b_{0}$ is the relevant scaling parameter [23]. After this stage of

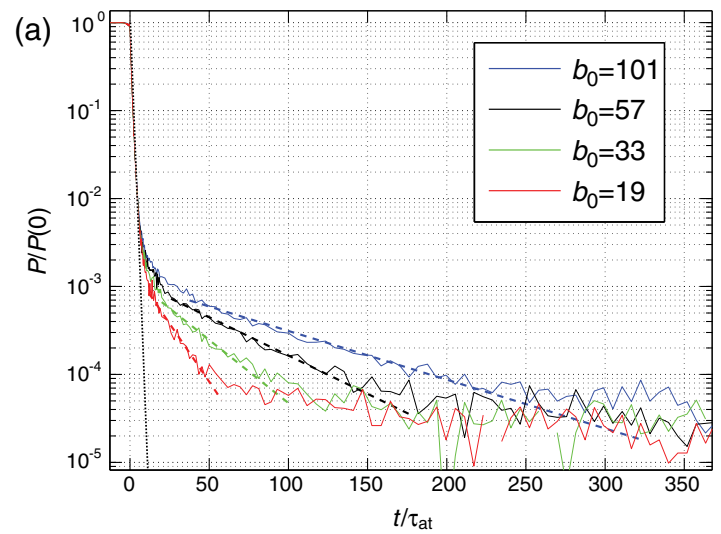

expansion and measurement, the MOT is switched on again and most of the atoms are recaptured. The complete cycle is thus short enough to allow the signal integration over a large number of cycles, typically 500000 (complete acquisition time $\sim 14 \mathrm{~h}$ per run). The scattering of the probe beam is collected by a lens with a solid angle of $\sim 5 \times 10^{-2}$ sr at $\theta \approx 35^{\circ}$ from the incident direction of the laser beam (see Fig. 1). We use a hybrid photomultiplier (Hamamatsu HPM R10467U-50) in the photon-counting regime, without any measurable amount of afterpulsing, which would considerably mask signatures of subradiance. The signal is then recorded on a multichannel scaler (MCS6A by FAST ComTec) with a time bin of $1.6 \mathrm{ns,}$ averaging over the cycles. The cooperativity parameter $b_{0}$ corresponding to each pulse is calibrated by an independent measurement of the atom number, cloud size, and temperature using absorption imaging [23].

Typical data are shown in Fig. 2. The signal is normalized to the steady-state fluorescence level and we focus on the switch-off period to highlight the slow fluorescence decay. In Fig. 2(a), the detuning $\delta$ of the probe beam is kept constant and the different decay curves correspond to different values of $b_{0}$, obtained in a single run. On the contrary, Fig. 2(b) shows data taken with different detunings but for the same $b_{0}$. In both cases, most of the fluorescence decays fast (note the logarithmic scale of the vertical axis), but a slow decay is clearly seen well above the noise floor (slightly below $10^{-4}$ ). We stress that fluorescence can be detected at very large delays, as can be seen from the time axis, in units of $\tau_{\mathrm{at}}=\Gamma^{-1}=26 \mathrm{~ns}$. We attribute this slow decay to subradiance in the single-photon (or weak excitation) regime, as predicted in Ref. [22].

A qualitative analysis of the two figures clearly shows different behaviors. As $b_{0}$ is varied, the slow decay rate changes, whereas its relative amplitude stays

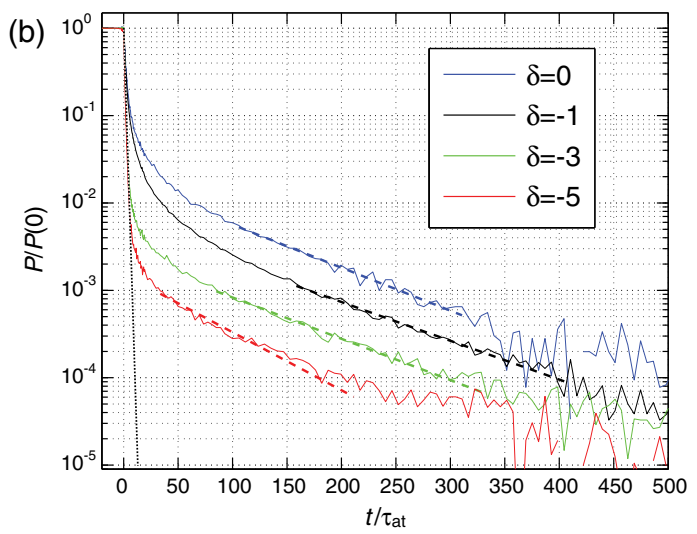

FIG. 2. Slow decay of the fluorescence after switching off the probe laser. The vertical scale is normalized by the steady-state detected power $P(0)$, and the time scale is normalized by the atomic lifetime of the excited state $\tau_{\mathrm{at}}$. Without any collective effect (single-atom physics), the decay would be given by $P(t)=P(0) \exp \left(-t / \tau_{\text {at }}\right)$ (the black dotted line). (a) Several data are shown for different onresonance optical depths $b_{0}$ and the same detuning $\delta=-6$ (in units of $\Gamma$ ). The time constant increases with $b_{0}$. (b) Several data are shown for different detunings and the same $b_{0}=108 \pm 5$. The time constant remains unchanged, but the relative amplitude of the subradiant decay decreases as the detuning increases. 
approximately the same. The exact opposite happens when we change the detuning, keeping $b_{0}$ fixed. For a quantitative analysis, we fit the slow tail at long delays by an exponential decay with two free parameters: the time constant $\tau_{\text {sub }}$ and its relative amplitude $A_{\text {sub }}$ [23]. We systematically studied how these parameters depend on $b_{0}$ and $\delta$. The result of this analysis is presented in Fig. 3. In Fig. 3(a) we plot the subradiant time constant as a function of $b_{0}$ for different detunings. The collapse of all points on the same curve clearly indicates that the slow decay rate does not depend on the detuning [see also Fig. 3(b)]. This demonstrates that this slow decay is not a multiplescattering effect, such as previously observed radiation trapping [36], which depends on the optical depth at the laser frequency $b(\delta) \propto b_{0} /\left(1+4 \delta^{2}\right)$, with a strong dependance with $\delta$. The second feature in Fig. 3(a) is the linear increase of $\tau_{\text {sub }}$ with $b_{0}$, up to time constants as long as $\tau_{\text {sub }} \sim 100 \tau_{\text {at }}$. This is perfectly consistent with the predictions of the coupled-dipole model for subradiance [23]. We note that for large negative detunings, one has to take into account the variation of $b_{0}$ during the pulse series induced by the cloud expansion together with a significant contribution of atom losses by off-resonant hyperfine pumping. This allowed us to test different combinations of $N$ and $R$ as scaling parameters (see Fig. 2 of the Supplemental Material [23]). The comparison showed that the best collapse has been obtained with $N / R^{2} \propto b_{0}$ as the scaling parameter, which demonstrates that $b_{0}$ is indeed the relevant cooperativity parameter in the regime of a dilute and extended sample, as expected from the ratio of the number of atoms to the number of available electromagnetic modes radiating from the sample. Finally, we show in Fig. 3(c) the relative amplitude $A_{\text {sub }}$ of the slow decay. As was already seen in Fig. 2(b), this amplitude is much larger near resonance, and it seems to reach a plateau for large detunings. We have checked that subradiant decay is still visible at a larger detuning, up to $\delta=-11$ [23]. This is in line with the coupled-dipole model, in which the weight of the long-lived modes are enhanced near resonance and the weight of all of the collective modes becomes independent of the detuning at large detuning.

As a long lifetime can also occur due to multiple scattering, when the optical depth $b(\delta) \gg 1$ [36], we investigated the decay time close to the atomic resonance to study how subradiance compares with radiation trapping. In the range of the accessible experimental values [Fig. 3(b)], no marked difference of the decay times around resonance is visible, even though a small difference was predicted in Ref. [22]. The interplay of radiation trapping and subradiance near resonance is still an open question and will be the subject of our further experiments.

We have also studied the effect of the probe intensity and checked that, at low saturation parameter, the observed subradiance is independent of the intensity (see Fig. 3 of the Supplemental Material [23]), which validates the use of the coupled-dipole model in the weak-excitation limit. We finally also excluded the possibility that residual
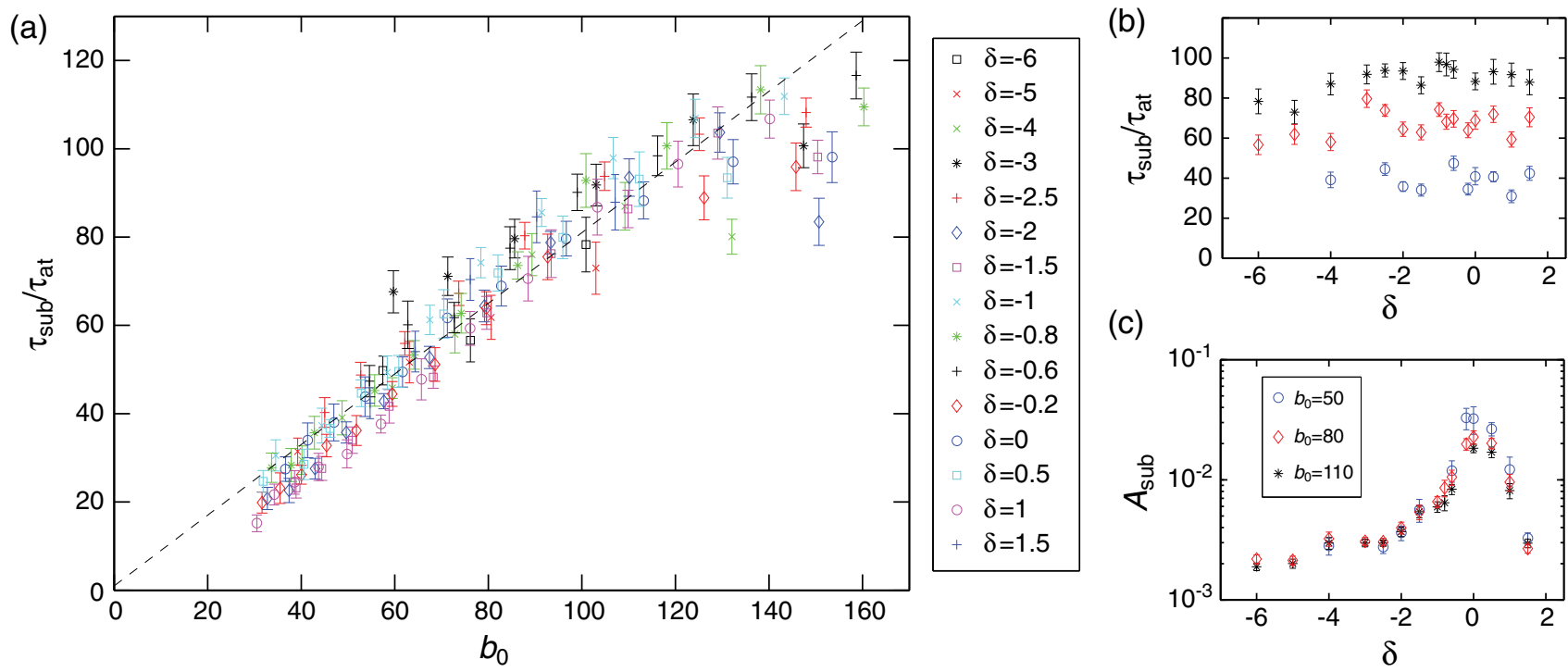

FIG. 3. Dependence of the subradiance time with the on-resonance optical depth $b_{0}$ and the laser detuning $\delta$. (a) Subradiant time constant $\tau_{\text {sub }}$ (in units of $\tau_{\text {at }}$ ) as a function of $b_{0}=3 N /\left(k_{0} R\right)^{2}$ for different detunings of the probe laser. Almost all of the points collapse on a single curve, showing the linear scaling with $b_{0}$. The dashed line is a linear fit $\tau_{\text {sub }} / \tau_{\text {at }}=1+\alpha b_{0}$, with the slope $\alpha$ as a free parameter. Excluding the data with $b_{0}>120$, we obtain $\alpha=0.8$. (b) From the same data, $\tau_{\text {sub }}$ is plotted as a function of the detuning for three different values of $b_{0}$, illustrating that $\tau_{\text {sub }}$ is independent of the detuning. (c) Similarly, the subradiant relative amplitude $A_{\text {sub }}$ is plotted as a function of the detuning for the same three different values of $b_{0}$. Subradiance is more important near resonance and decreases towards a plateau at large detuning. 
near-resonant light might always be present and induce a slow decay due to radiation trapping, thus mimicking off-resonant subradiance [23].

To summarize, we have presented the first direct signatures of subradiance in a large system of resonant scatterers. We have shown that in the regime of dilute and extended samples, the subradiant decay rate is governed by a cooperativity parameter defined as the ratio of the number of scatterers and the sample size squared, which conveniently corresponds to the on-resonance optical depth. This observation of subradiance opens interesting questions, including the robustness of subradiance against decoherence mechanisms or the possibility of controlling the population of the subradiant modes by an appropriate temporal or spatial shaping of the driving laser or of the atomic levels. If the subradiant states can be manipulated with sufficient control [37], their isolation from the environment might be exploited as a resource for quantum information or quantum metrology [38]. As subradiance goes hand in hand with superradiance, simultaneous recording of fast and slow decays would be a beautiful illustration of the cooperative scattering envisioned by Dicke. By using a stronger laser drive, it would also be possible to access a larger subspace of the full Hilbert space, addressing the possibility of a photon-blockade effect [39].

In addition to quantum optics, our observation is also relevant to mesoscopic physics [40,41], a community less familiar with Dicke physics. One major challenge in this field is the observation of strong localization of light, the analogy for classical waves of Anderson localization of electrons [42]. Previous experimental observations of a decay of scattered light slower than predicted by the diffusion equation have been used as a signature of Anderson localization in dielectrics $[43,44]$. Our results show that it cannot always be the case with cold atoms. Similarly, recent numerical simulations considering pointdipole resonant scatterers study the collective modes of the effective Hamiltonian of the system and, in particular, their lifetimes [45-49]. Our work shows that Dicke subradiance can also be at the origin of very long lifetimes and that a careful analysis is required to distinguish subradiant from localized modes [49]. Finally, the combination of subradiance with disorder acting on the atomic transitions might provide an alternative route to strong localization of light, as was recently suggested [50].

We acknowledge fruitful discussions with Nicola Piovella, Tom Bienaimé, Romain Bachelard, Guillaume Labeyrie, and Dominique Delande and the technical help from Louis Bellando, Ivor Kresic, Loïc Lavenu, and Antoine Dussaux, and we thank Alain Aspect for his constructive comments on the manuscript. We also acknowledge financial support from the French Agence National pour la Recherche (project LOVE, Grant No. ANR-14-CE260032), the Brazilian Coordenaçáo de Aperfeiçoamento de Pessoal de Nível Superior (CAPES), the Brazilian
Conselho Nacional de Desenvolvimento Científico e Tecnológico (project PVE, Grant No. 303426/2014-4), and the European Research Executive Agency (program COSCALI, Grant No. PIRSES-GA-2010-268717).

*william.guerin@inln.cnrs.fr

[1] Q. Baudouin, W. Guerin, and R. Kaiser, in Annual Review of Cold Atoms and Molecules, Vol. 2, edited by K. Madison, K. Bongs, L. D. Carr, A. M. Rey, and H. Zhai (World Scientific, Singapore, 2014), p. 251.

[2] G. Labeyrie, Mod. Phys. Lett. B 22, 73 (2008).

[3] R. Kaiser, J. Mod. Opt. 56, 2082 (2009).

[4] N. Sangouard, C. Simon, H. de Riedmatten, and N. Gisin, Rev. Mod. Phys. 83, 33 (2011).

[5] R. H. Dicke, Phys. Rev. 93, 99 (1954).

[6] N. Skribanowitz, I. P. Herman, J. C. MacGillivray, and M. S. Feld, Phys. Rev. Lett. 30, 309 (1973).

[7] R. Friedberg, S. R. Hartmann, and J. T. Manassah, Phys. Rep. 7, 101 (1973).

[8] M. Gross and S. Haroche, Phys. Rep. 93, 301 (1982).

[9] D. Pavolini, A. Crubellier, P. Pillet, L. Cabaret, and S. Liberman, Phys. Rev. Lett. 54, 1917 (1985).

[10] R. G. DeVoe and R. G. Brewer, Phys. Rev. Lett. 76, 2049 (1996).

[11] M. D. Barnes, P. S. Krstic, P. Kumar, A. Mehta, and J. C. Wells, Phys. Rev. B 71, 241303(R) (2005).

[12] B. H. McGuyer, M. McDonald, G. Z. Iwata, M. G. Tarallo, W. Skomorowski, R. Moszynski, and T. Zelevinsky, Nat. Phys. 11, 32 (2015).

[13] V. V. Temnov and U. Woggon, Phys. Rev. Lett. 95, 243602 (2005)

[14] M. J. Stephen, J. Chem. Phys. 40, 669 (1964).

[15] M. O. Scully, E. S. Fry, C. H. Raymond Ooi, and K. Wódkiewicz, Phys. Rev. Lett. 96, 010501 (2006).

[16] M. O. Scully and A. A. Svidzinsky, Science 325, 1510 (2009).

[17] R. Röhlsberger, K. Schlage, B. Sahoo, S. Couet, and R. Rüffer, Science 328, 1248 (2010).

[18] A. A. Svidzinsky, J.-T. Chang, and M. O. Scully, Phys. Rev. Lett. 100, 160504 (2008).

[19] A. Svidzinsky and J.-T. Chang, Phys. Rev. A 77, 043833 (2008).

[20] R. A. de Oliveira, M. S. Mendes, W. S. Martins, P. L. Saldanha, J. W. R. Tabosa, and D. Felinto, Phys. Rev. A 90, 023848 (2014).

[21] P. Longo, C. H. Keitel, and J. Evers, arXiv:1503.04532.

[22] T. Bienaimé, N. Piovella, and R. Kaiser, Phys. Rev. Lett. 108, 123602 (2012).

[23] See Supplemental Material at http://link.aps.org/ supplemental/10.1103/PhysRevLett.116.083601, which includes Refs. [24-35], for the details of the calibration of the optical depth and data analysis, a brief presentation, and the main predictions of the coupled-dipole model, the test of different scaling parameters, the data taken for different probe intensities, and the analysis of the possible role of radiation trapping of near-resonant light.

[24] P. W. Courteille, S. Bux, E. Lucioni, K. Lauber, T. Bienaimé, R. Kaiser, and N. Piovella, Eur. Phys. J. D 58, 69 (2010). 
[25] A. A. Svidzinsky, J.-T. Chang, and M. O. Scully, Phys. Rev. A 81, 053821 (2010).

[26] T. Bienaimé, M. Petruzzo, D. Bigerni, N. Piovella, and R. Kaiser, J. Mod. Opt. 58, 1942 (2011).

[27] T. Bienaimé, R. Bachelard, P. W. Courteille, N. Piovella, and R. Kaiser, Fortschr. Phys. 61, 377 (2013).

[28] Y. Miroshnychenko, U. V. Poulsen, and K. Mølmer, Phys. Rev. A 87, 023821 (2013).

[29] W. Feng, Y. Li, and S.-Y. Zhu, Phys. Rev. A 89, 013816 (2014).

[30] G. Labeyrie, R. Kaiser, and D. Delande, Appl. Phys. B 81, 1001 (2005).

[31] C. Cohen-Tannoudji, J. Dupont-Roc, and G. Grynberg, Atom-Photon Interactions: Basic Processes and Applications (Wiley, New York, 1992).

[32] B. R. Mollow, Phys. Rev. 188, 1969 (1969).

[33] See, e.g., T. Chanelière, Ph.D. thesis, Université Nice Sophia Antipolis, 2004, p. 40, https://tel.archives-ouvertes .fr/tel-00007793.

[34] S. Kraft, A. Deningen, C. Trück, J. Fortágh, F. Lison, and C. Zimmermann, Laser Phys. Lett. 2, 71 (2005).

[35] R. Pierrat, B. Grémaud, and D. Delande, Phys. Rev. A 80, 013831 (2009).

[36] G. Labeyrie, E. Vaujour, C. A. Müller, D. Delande, C. Miniatura, D. Wilkowski, and R. Kaiser, Phys. Rev. Lett. 91, 223904 (2003).

[37] M. O. Scully, Phys. Rev. Lett. 115, 243602 (2015).
[38] L. Ostermann, H. Ritsch, and C. Genes, Phys. Rev. Lett. 111, 123601 (2013).

[39] J. Pellegrino, R. Bourgain, S. Jennewein, Y. R. P. Sortais, A. Browaeys, S. D. Jenkins, and J. Ruostekoski, Phys. Rev. Lett. 113, 133602 (2014).

[40] M. C. W. van Rossum and T. M. Nieuwenhuizen, Rev. Mod. Phys. 71, 313 (1999).

[41] E. Akkermans and G. Montambaux, Mesoscopic Physics of Electrons and Photons (Cambridge University Press, Cambridge, England, 2007).

[42] P. W. Anderson, Phys. Rev. 109, 1492 (1958).

[43] C. M. Aegerter, M. Störzer, and G. Maret, Europhys. Lett. 75, 562 (2006).

[44] F. Scheffold and D. Wiersma, Nat. Photonics 7, 934 (2013).

[45] E. Akkermans, A. Gero, and R. Kaiser, Phys. Rev. Lett. 101, 103602 (2008).

[46] S. E. Skipetrov and I. M. Sokolov, Phys. Rev. Lett. 112, 023905 (2014).

[47] L. Bellando, A. Gero, E. Akkermans, and R. Kaiser, Phys. Rev. A 90, 063822 (2014).

[48] S. E. Skipetrov and I. M. Sokolov, Phys. Rev. Lett. 114, 053902 (2015).

[49] C. E. Máximo, N. Piovella, P. W. Courteille, R. Kaiser, and R. Bachelard, Phys. Rev. A 92, 062702 (2015).

[50] A. Biella, F. Borgonovi, R. Kaiser, and G. L. Celardo, Europhys. Lett. 103, 57009 (2013). 